\title{
Fabrication of Poly(Glycerol Sebacate)-Poly( $\varepsilon$-Caprolactone) Extrusion-Based Scaffolds for Cartilage Regeneration
}

\author{
D. Reis ${ }^{1, a}$, S. Biscaia ${ }^{1, b}$, I.J. Seabra ${ }^{2, c}$, A. Veloso ${ }^{3, d}$, and P. Morouço $0^{1, e^{*}}$
}

\begin{abstract}
${ }^{1}$ Centre for Rapid and Sustainable Product Development, Polytechnic Institute of Leiria, Portugal,
${ }^{2}$ College of Agriculture, Polytechnic Institute of Coimbra, Portugal,

${ }^{3}$ Coimbra Institute of Engineering, Polytechnic Institute of Coimbra, Portugal.

adiana.reis@ipleiria.pt, bsara.biscaia@ipleiria.pt, ciseabra@esac.pt, danaveloso@isec.pt, epedro.morouco@ipleiria.pt
\end{abstract}

Keywords: Biofabrication, Bioprinting, Poly (glycerol sebacate), Tissue engineering.

\begin{abstract}
Cartilage related diseases are on the top list concerns of the World Health Organization, being the prevention of articular cartilage degeneration a major health matter for which there are few effective solutions. Using an extrusion-based approach and a polyester elastomer it was aimed to produce $3 \mathrm{D}$ structures with controlled architecture and with closer mimicry to cartilage native tissue. The obtained constructs demonstrated high reliability, being the addition of poly (glycerol sebacate) a procedure to enhance the properties of the constructs.
\end{abstract}

\section{Introduction}

Osteoarthritis (OA) is a chronic joint condition affecting over 250 million people worldwide [1]. Along with a significant impact on health-care and society, the Global Burden of Disease Study from the World Health Organization reported that knee OA is the 11th leading cause of disability, and shows a growing trend [2]. Although it may damage every joint in the human body, the most common disorders affect joints in the hips, knees, hands and spine. Thus, with a society facing a demographic transformation, with an epidemic increase of obesity, integrated interventions to weaken the problem are mandatory. Each year, more than 50 million people visit doctors because of joint pain; half of them with a damage of the articular cartilage.

Apart from not being possible to reverse the underlying process, in its early stages OA symptoms can usually be effectively managed. In fact, adopting a healthy lifestyle may slow the disease progression, and help improve joint function and reduce pain [3]. On the other hand, in the following stages, surgery may be required. Thus, significant efforts are being developed worldwide in the fields of tissue engineering and regenerative medicine, but full cartilage restoration remains a paramount challenge [4]. Tissue engineering is a multidisciplinary scientific field, which applies a wide variety of methodologies. Therefore, multidisciplinary research teams can provide suitable inputs for its development [5,6]. One of the major goals is to produce biological substitutes to restore, maintain or improve tissue function, using biocompatible and biodegradable support structures, i.e. scaffolds, in conjunction with human cells [7].

Cartilage is a tissue with a huge complexity, which is present in the human body in three types: hyaline cartilage, fibrocartilage and elastic cartilage. Apart some resemblances, these types are quite different and play unlike roles for human functionality. For instance, the hyaline cartilage, also known as articular cartilage, has a major role in providing joints with a surface that combines low friction with high lubrication [8]. A deeper knowledge on cartilage characterization, bridging the gap between anatomy and physiology, may lead the way for better implants aiming cartilage repair and regeneration [9]. This is of even more interest as cartilage is an avascular tissue of the human body, hence with an extremely low capability for tissue regeneration. Regardless of the low metabolic activity and relatively poor ability to heal of chondrocytes, hyaline cartilage is a dynamic and responsive tissue, where the contribution of cell produced extracellular matrix components play a major role [10]. It is well documented in the literature that hyaline cartilage has remarkable 
mechanical properties (elastic modulus of $\sim 123 \mathrm{MPa}$; mechanical tensile strength of $17 \mathrm{MPa}$; compressive modulus varying between 0.53 and $1.82 \mathrm{MPa}$; and compressive stress between 14-59 MPa) [11,12] and lasting durability, despite its few millimetres of thickness. The referred complexity and properties demonstrate the challenges faced by research groups aiming to fully restore cartilage functionality.

The interest in elastomeric biomaterials has been significantly increasing as they offer the possibility to engineer implantable structures resembling the elasticity of native tissues. Poly (glycerol-sebacate) (PGS) is a polyester elastomer originally synthesized by Langer's group and widely explored for soft tissue engineering. Despite its useful properties of biocompatibility and in vivo biodegradation, the complex processing conditions remain the main difficulty to extend the range of applications. In addition, current biofabrication strategies for PGS-based biomaterials are limited to conventional processes (e.g. freeze drying, melt moulding, electrospinning), which prevent the fabrication of patient-specific constructs with controlled architectures, interconnectivity between pores, porosity and pore size.

So, the main aim of the present work was to examine the feasibility for producing extrusionbased scaffolds using PGS. It was hypothesized that scaffolds with incorporation of PGS would demonstrate properties resembling the cartilage native tissue.

\section{Materials and Methods}

\section{Materials}

Commercial PGS (Regenerez $\left.{ }^{\circledR}\right)$ was obtained from Sigma-Aldrich. PCL polymer (CAPA® 6500, Mw: 50,000 Da) was purchased from Perstorp Caprolactones (Cheshire, UK). Mixtures were prepared using chloroform (analytical grade) from Scharlau Chemie (Barcelona, Spain).

\section{Fabrication of 3D scaffolds}

PGS-PCL and PCL membranes were prepared by solvent casting, to obtain 3D scaffolds through an additive manufacturing system. PCL pellets were dissolved in chloroform at $50^{\circ} \mathrm{C}$ and the solution was deposited in Petri dishes and dried at room temperature. The PGS-PCL mixture was prepared through the dissolution of PCL pellets $(50 \%(\mathrm{w} / \mathrm{w}))$ and PGS $50 \%(\mathrm{w} / \mathrm{w})$ in chloroform at $50^{\circ} \mathrm{C}$. After obtaining a homogeneous solution, the PGS-PCL mixture was deposited in Petri dishes and dried at room temperature.

Scaffolds were produced by using a layer-by-layer manufacturing system [13]. All 3D structures were prepared with an inter-filament distance of $650 \mu \mathrm{m}$, nozzle diameter of $300 \mu \mathrm{m}$ and $0^{\circ} / 90^{\circ}$ pore configuration. Control PCL and PGS-PCL scaffolds were obtained using the same processing conditions (deposition velocity of $5 \mathrm{~mm} / \mathrm{s}$ and liquefier temperature of $80^{\circ} \mathrm{C}$ ), with $10 \mathrm{rpm}$ of screw rotation velocity.

\section{Characterization of 3D scaffolds}

The surface topographies of scaffolds were examined by optical microscope Daffodil MCX100 (Micros Austria) connected to a digital camera at a magnification of 40x.

The thermal properties of the samples were evaluated with a STA 6000 (Perkin Elmer). Samples of 6-7 mg were placed in alumina pans and empty pans were used as reference. All samples were first heated at a range of $15-120^{\circ} \mathrm{C}$ at a heating rate of $10^{\circ} \mathrm{C} / \mathrm{min}$ and held isothermally for $5 \mathrm{~min}$ to mitigate any prior thermal history. Then, the samples were cooled to $15^{\circ} \mathrm{C}$ at $10^{\circ} \mathrm{C} / \mathrm{min}$, held isothermally for $10 \mathrm{~min}$ and then reheated to $160{ }^{\circ} \mathrm{C}$ at the same rate. After each test, the melting point region from the thermograph was analysed to determine the heat of fusion $\left(\Delta H_{f u s}\right)$ and the melting temperature $\left(T_{m}\right)$; the crystallization region was analysed to determine the crystallization temperature $\left(T_{c}\right)$ of all samples. To evaluate the thermal degradation, the samples were exposed to a temperature ramp from $15{ }^{\circ} \mathrm{C}$ to $600{ }^{\circ} \mathrm{C}$, at a heating rate of $10{ }^{\circ} \mathrm{C} / \mathrm{min}$. Initial degradation temperature $\left(T D_{o n}\right)$ and peak degradation temperature $\left(T D_{p}\right)$ were determined using the first 
derivative curve to analyse the thermal degradation of the specimens. The flow rate of nitrogen was $20 \mathrm{~mL} / \mathrm{min}$ during all the runs.

FT-IR analyses of all samples were performed with an ATR Fourier transform infrared spectrometer (Alpha FT-IR spectrometer, Bruker, Belgium). The spectra were averaged over 64 scans at a resolution of $4 \mathrm{~cm}^{-1}$. All tests were performed in triplicate.

\section{Results}

\section{Development and characterization of PGS-PCL scaffolds}

In Figure 1 the PGS-PCL 3D structures are presented. It was possible to obtain a controlled architecture with a porous structure, and with remarkable flexibility. Micrographs (Figure 2) were assessed to explore the alignment and orientation of the filaments, and their morphological characteristics (Table 1), both with and without PGS.
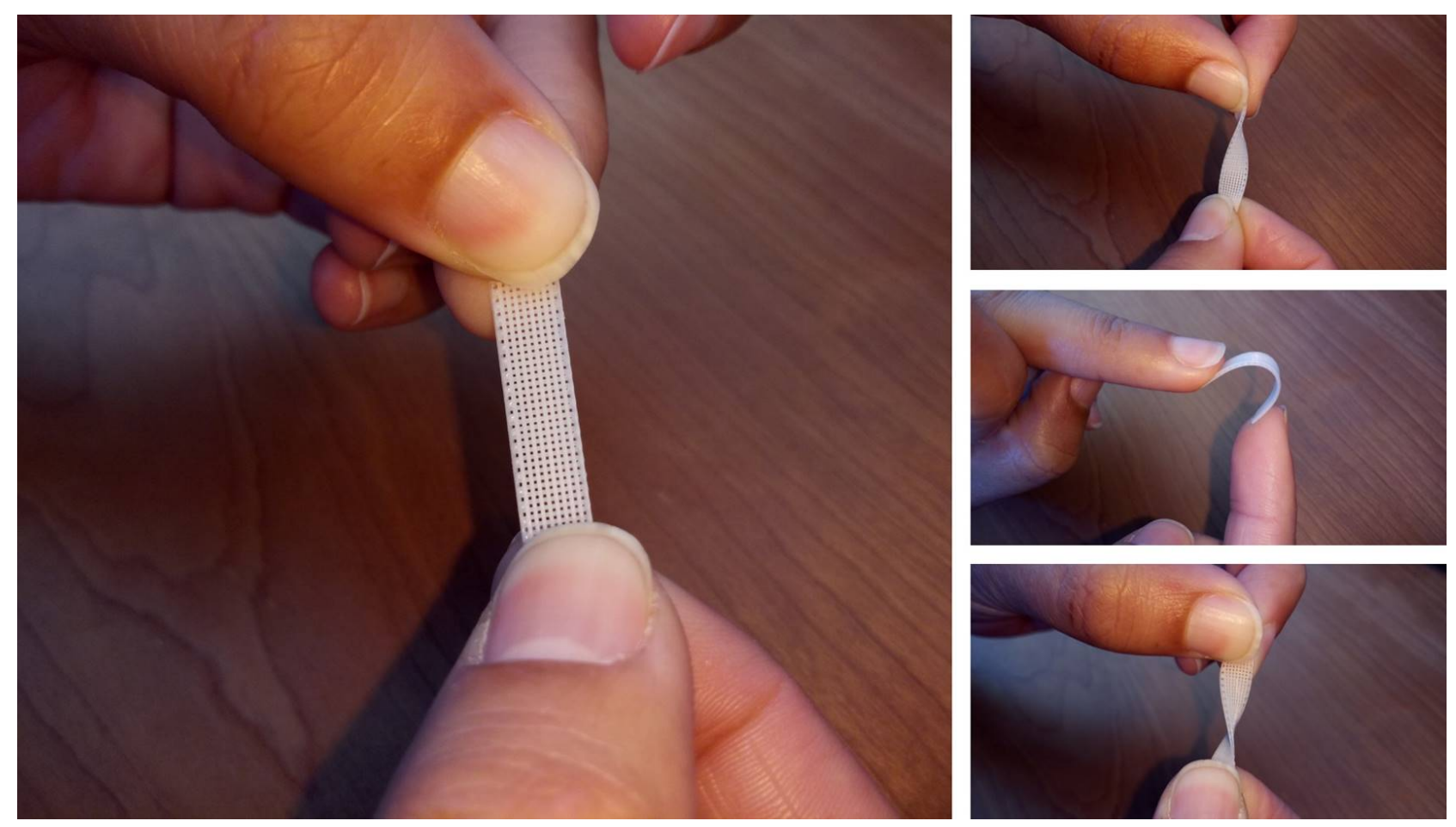

Figure 1.3D structures of PCL/PGS
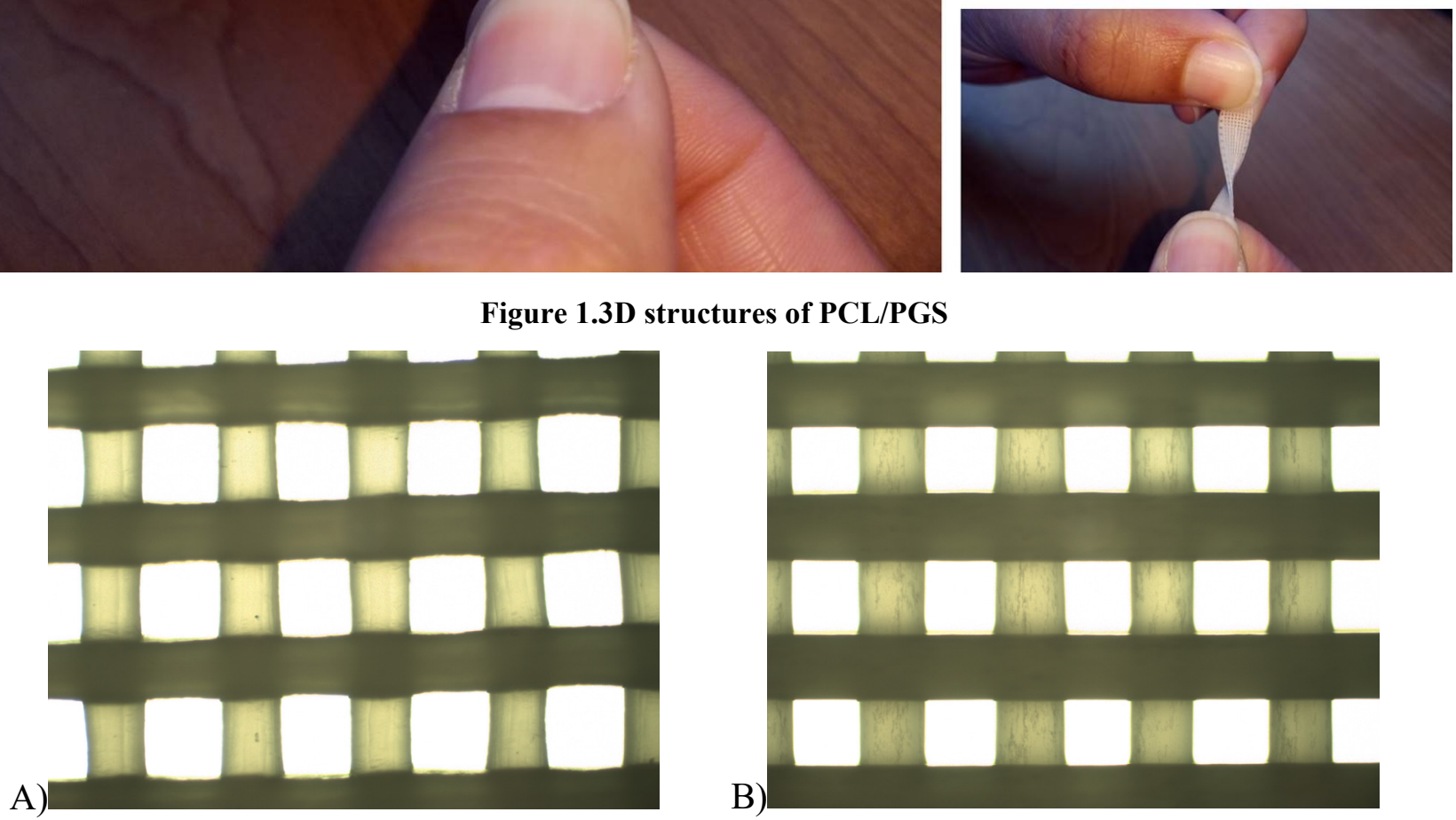

Figure 2. Micrographs (magnification: 40x) of PCL (a) and PGS-PCL (b) scaffolds 
Table 1. Scaffolds morphological values $($ mean $\pm \mathbf{s d})$

\begin{tabular}{lcc}
\hline Scaffold & Filament diameter $(\boldsymbol{\mu m})$ & Pore size $(\boldsymbol{\mu m})$ \\
\hline $\boldsymbol{P C L}$ & $274.3 \pm 3.1$ & $313.7 \pm 4.5$ \\
$\boldsymbol{P G S}$-PCL & $275.0 \pm 4.6$ & $303.0 \pm 3.0$ \\
\hline
\end{tabular}

\section{Thermal behaviour}

The thermal properties were studied by DSC and TGA (Figure 3) and the thermal parameters are summarized in Table 2.
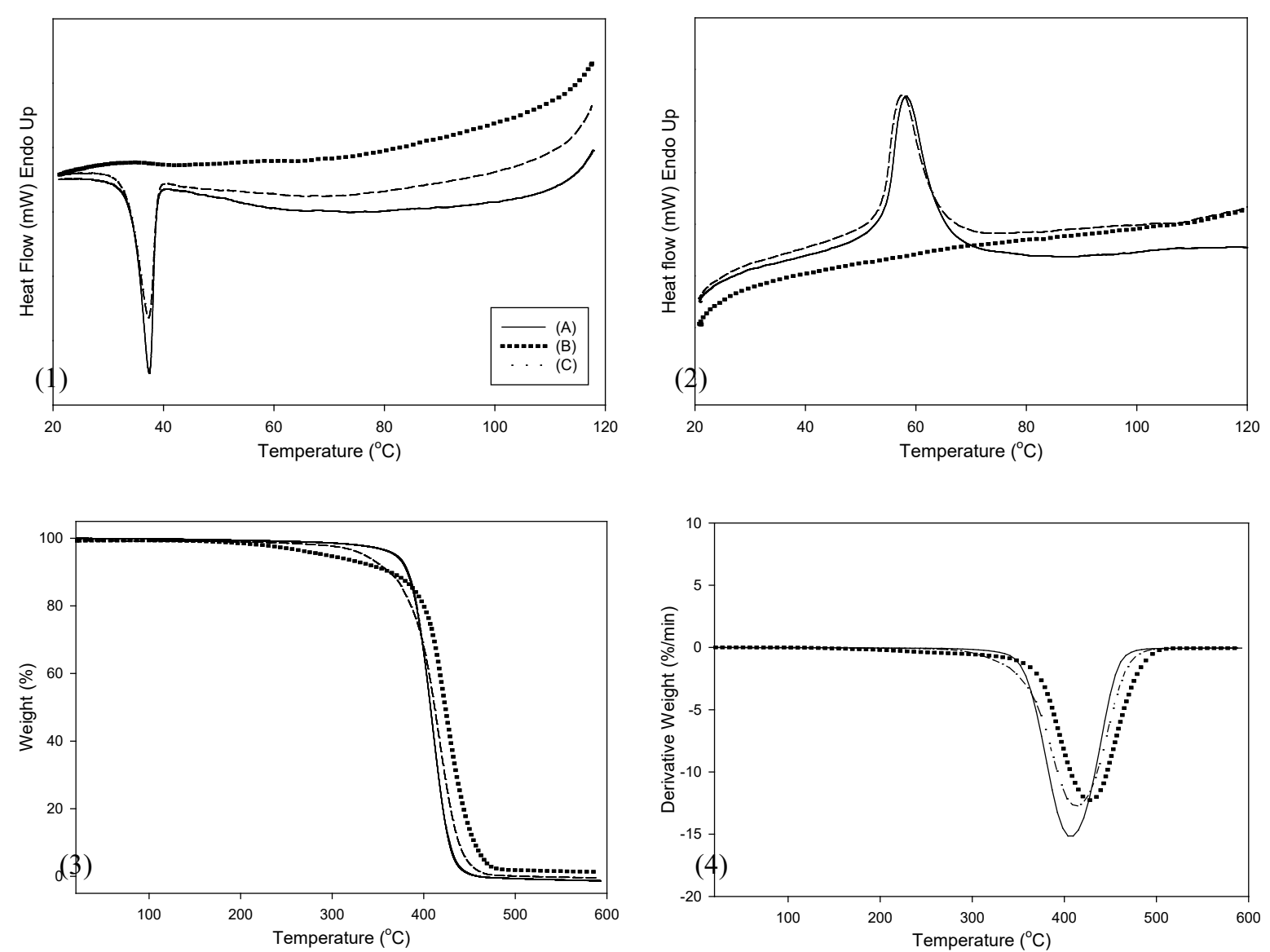

Figure 3. Thermal properties of: (A) PCL, (B) PGS and (C) PCL/PGS. (1) DSC thermograph: cooling cycle, (2) DSC thermograph: $2^{\text {nd }}$ heating cycle, (3) TGA thermograph and (4) DTG thermograph

Table 2. Thermal properties of the samples (mean $\pm \mathrm{sd}$ )

\begin{tabular}{lcccccc}
\hline & & DSC & TGA & \multicolumn{2}{c}{ DTG } \\
& $T_{c}(\circ \mathbf{\circ})$ & $T_{m}(\circ \mathbf{\circ})$ & $\Delta H f_{u s}(\mathbf{J} / \mathbf{g})$ & Mass loss $(\%)$ & $T_{\text {on }}(\circ \mathbf{C})$ & $\boldsymbol{T D}_{\boldsymbol{p}}(\circ \mathbf{\circ})$ \\
\hline PCL & $37.28 \pm 0.29$ & $58.30 \pm 0.18$ & $50.79 \pm 2.80$ & $99.32 \pm 0.40$ & $384.84 \pm 2.93$ & $410.07 \pm 0.61$ \\
PGS & $*$ & $*$ & $*$ & $96.88 \pm 0.70$ & $404.04 \pm 7.37$ & $422.76 \pm 3.19$ \\
PGS-PCL & $37.30 \pm 0.05$ & $57.40 \pm 0.19$ & $41.56 \pm 3.01$ & $98.13 \pm 0.42$ & $385.63 \pm 3.89$ & $412.91 \pm 3.72$ \\
\hline
\end{tabular}




\section{Chemical characterization}

In Figure 4 the FTIR spectra of PGS-PCL and PCL membrane are presented.

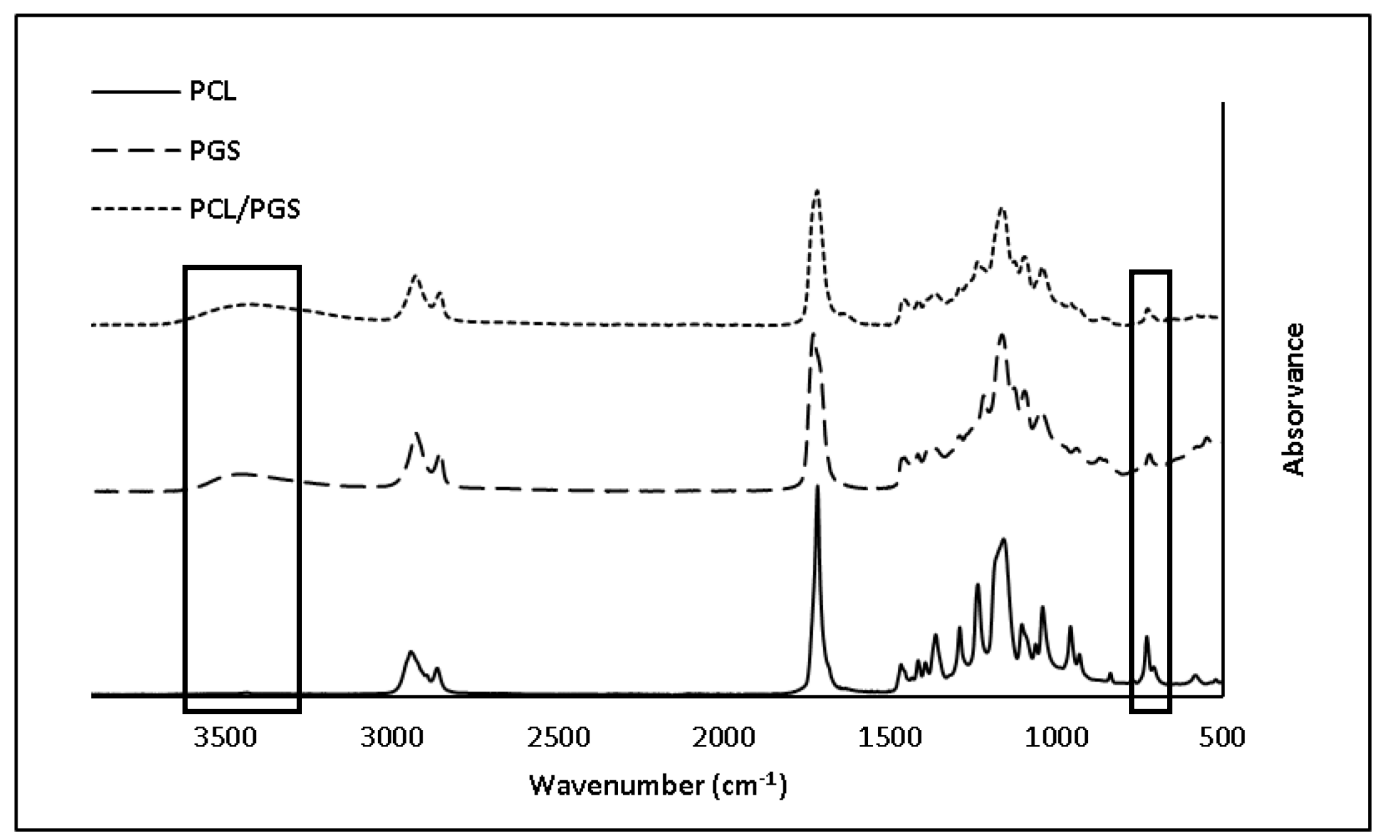

Figure 4. FTIR-ATR spectra of PCL, PGS and PGS-PCL samples

\section{Discussion}

Gathering tissue engineering and regenerative medicine, researchers have been interested in developing alternative approaches for restoring joint functionality. For instance, the creation of constructs with a structure and composition resembling native cartilage and yielding similar mechanical behaviour [14]. One of the most promising methodologies is the use of additive manufacturing (AM) processes. AM technologies allow the production of complex 3D structures with a high level of control, predefined geometry, size and interconnected pores, in a reproducible way. This controlled organization enhances the vascularization and, thus, transport of oxygen and nutrients throughout the whole structure, providing an adequate biomechanical environment for tissue regeneration [15]. However, adapting the adequate technology with enhanced biomaterials to obtain customized implants that mimic the native tissue, remains an utmost challenge. The present study produced PGS-PCL scaffolds with controlled architecture. The constructs presented high reliability (coefficient of variation lower than $2 \%$ ), demonstrating the feasibility of having elastomer-based scaffolds by an extrusion process.

Biodegradable and biocompatible elastomeric biomaterials are appealing for tissue engineering and drug delivery, as they resemble the elasticity of native extracellular matrix network. Such materials also have the ability to recover from medium to large deformations with minimal irritation to the surrounding tissues, while maintain the original function. PGS is a polyester elastomer developed by the Langer's group through a polycondensation reaction of glycerol and sebacic acid, yielding to a biocompatible and biodegradable elastomeric material. Experimental results showed that PGS implants are totally absorbed in vivo after 60 days of implantation, contrasting with the weight loss of $17 \pm 6 \%$ after 60 days of in vitro degradation in PBS [16]. Importantly, the degradation products of PGS are eliminated through natural pathways [17].

Regarding DSC results, experiments with pure PGS were also done. However, it was not possible to observe the melting and crystallization temperatures in the heating and cooling cycles performed, respectively, which was due to a limitation of the instrument used in the study. In previous works Gaharwar et al. [18] and Zhao et al. [19] calculated the melting temperature at $\approx 10{ }^{\circ} \mathrm{C}$. On the other hand, Wang et al. [16] revealed two crystallization temperatures at $-52.14{ }^{\circ} \mathrm{C}$ 
and $-18.50{ }^{\circ} \mathrm{C}$. PCL $T_{c}$ and $T_{m}$ were in agreement with the previously published reports $[13,14$, 17], and the melting enthalpy was calculated to be $50.79 \mathrm{~J} / \mathrm{g}$. PGS-PCL scaffold exhibited a small decrease of melting temperature, which can be related to a possible miscibility of PGS and PCL and/or the presence of the other polymer $[14,17]$. The weight loss characteristic of PCL, PGS and PGS-PCL was evaluated by thermal gravimetric analysis (TGA) (Figure 3 (3)). The derivative curve of the thermal decomposition spectra of all the samples indicates a step degradation profile and was calculated to evaluate the onset degradation temperature and peak degradation temperature. The addition of PGS induced a minimal increase in $T D_{o n}$ and $T D_{p}$, suggesting that adding PGS within the PCL network enhances the thermal stability, when compared with pure PCL.

PCL exhibited alkyl groups at 2943 and $2864 \mathrm{~cm}^{-1}$, and the strongest band at $1720 \mathrm{~cm}^{-1}$ which represent a carbonyl stretching, due to the presence of an ester in its composition. The spectra of PGS shows the same peaks for alkyl groups and peaks at $1720 \mathrm{~cm}^{-1}$ attributed to the presence of ester and carbonyl groups $(\mathrm{C}=\mathrm{O})$. There is also a band between 3600 and $3200 \mathrm{~cm}^{-1}$ for hydroxyl bond stretch vibration. In the PGS-PCL spectra, the main change observed was the broader hydroxyl signal at $3436 \mathrm{~cm}^{-1}$. According to Wang and co-workers [16], an intense $\mathrm{OH}$ stretch indicates that the hydroxyl groups are hydrogen bonded. The polymer surface is very hydrophilic because of the hydroxyl groups attached to its backbone. The peak at $1163 \mathrm{~cm}^{-1}$ is stronger in the PGS-PCL spectra than in the PCL spectra, and represents the C-O stretching in the crystalline phase [18]. Another difference is the peak at $730 \mathrm{~cm}^{-1}$ which is more intense in the PCL than PGS-PCL spectra, representing a stretching band of a methylene group [21].

\section{Conclusions}

In the last years, the world has assisted to an increase in the number of debilitating conditions and severe pain caused by cartilage defects, being the scientific and clinical community aware of the major problem that our society is facing. Regarding treatment, tissue science and regenerative medicine have emerged as promising disciplines concerning tissue and/or organ repair and regeneration. In fact, choosing the right approach for tissue regeneration is a major concern for every researcher in this field. The present study demonstrated that it is feasible to 3D print PGSPCL scaffolds, thus, enhancing their mechanical properties.

\section{Acknowledgements}

This research was supported by the European Regional Development Fund (FEDER), through COMPETE2020 under the PT2020 program (POCI-01-0145-FEDER-023423), by the Portuguese Foundation for Science and Technology (UID/Multi/04044/2013) and Centro2020 PAMI ROTEIRO/0328/2013 ( ${ }^{\circ}$ 022158).

\section{References}

[1] D.J. Hunter, D. Schofield, E. Callander, The individual and socioeconomic impact of osteoarthritis, Nat. Rev. Rheumatol. 10 (2014) 437-441.

[2] L.S. Lohmander, Knee replacement for osteoarthritis: facts, hopes, and fears, Medicographia 35 (2013) 181-188.

[3] C. Juhl, R. Christensen, E.M. Roos, W. Zhang, H. Lund, Impact of Exercise Type and Dose on Pain and Disability in Knee Osteoarthritis: A Systematic Review and Meta-Regression Analysis of Randomized Controlled Trials, Arthritis Rheumatol. 66 (2014) 622-636.

[4] T. Woodfield, K. Lim, P. Morouço, R. Levato, J. Malda, F. Melchels, Biofabrication in Tissue Engineering, in: Ref. Modul. Mater. Sci. Mater. Eng., 2017. 
[5] D.W. Hutmacher, Scaffolds in tissue engineering bone and cartilage, Biomaterials 21 (2000) 2529-2543.

[6] P.G. Morouço, N.M. Alves, S.C. Amado, The Role of Biomechanics in Tissue Engineering, Austin J Biomed Eng 3 (2016) 1035.

[7] J. Malda, J. Visser, F.P. Melchels, T. Jüngst, W.E. Hennink, W.J.A. Dhert, J. Groll, D.W. Hutmacher, 25th anniversary article: engineering hydrogels for biofabrication, Adv. Mater. 25 (2013) 5011-5028.

[8] D.R. Jeffrey, I. Watt, Imaging hyaline cartilage, Br. J. Radiol. (2014).

[9] V.H.M. Mouser, R. Levato, L.J. Bonassar, D.D. D’Lima, D.A. Grande, T.J. Klein, D.B.F. Saris, M. Zenobi-Wong, D. Gawlitta, J. Malda, Three-Dimensional Bioprinting and Its Potential in the Field of Articular Cartilage Regeneration, Cartilage (2016) 1947603516665445.

[10] M.I. Baker, S.P. Walsh, Z. Schwartz, B.D. Boyan, A review of polyvinyl alcohol and its uses in cartilage and orthopedic applications, J. Biomed. Mater. Res. Part B Appl. Biomater. 100 (2012) 1451-1457.

[11] T.D. Sargeant, A.P. Desai, S. Banerjee, A. Agawu, J.B. Stopek, An in situ forming collagenPEG hydrogel for tissue regeneration, Acta Biomater. 8 (2012) 124-132.

[12] H. Shin, B.D. Olsen, A. Khademhosseini, The mechanical properties and cytotoxicity of cellladen double-network hydrogels based on photocrosslinkable gelatin and gellan gum biomacromolecules, Biomaterials 33 (2012) 3143-3152.

[13] P. Morouço, S. Biscaia, T. Viana, M. Franco, C. Malça, A. Mateus, C. Moura, F.C. Ferreira, G. Mitchell, N.M. Alves, Fabrication of poly( $\epsilon$-caprolactone) scaffolds reinforced with cellulose nanofibers, with and without the addition of hydroxyapatite nanoparticles, Biomed Res. Int. 2016 (2016).

[14] L. Kock, C.C. van Donkelaar, K. Ito, Tissue engineering of functional articular cartilage: the current status, Cell Tissue Res. 347 (2012) 613-627.

[15] A.A. Zadpoor, J. Malda, Additive Manufacturing of Biomaterials, Tissues, and Organs, (2017).

[16] Y. Wang, G.A. Ameer, B.J. Sheppard, R. Langer, A tough biodegradable elastomer., Nat. Biotechnol. 20 (2002) 602-606.

[17] R.Z. Xiao, Z.W. Zeng, G.L. Zhou, J.J. Wang, F.Z. Li, A.M. Wang, Recent advances in PEGPLA block copolymer nanoparticles, Int. J. Nanomedicine 5 (2010) 1057-1065.

[18] A.K. Gaharwar, M. Nikkhah, S. Sant, A. Khademhosseini, Anisotropic poly (glycerol sebacate)-poly ( $\epsilon$-caprolactone) electrospun fibers promote endothelial cell guidance, Biofabrication 7 (2014) 15001.

[19] X. Zhao, Y. Wu, Y. Du, X. Chen, B. Lei, Y. Xue, P.X. Ma, A highly bioactive and biodegradable poly(glycerol sebacate)-silica glass hybrid elastomer with tailored mechanical properties for bone tissue regeneration, J. Mater. Chem. B 3 (2015) 3222-3233.

[20] S. Sant, D. Iyer, A.K. Gaharwar, A. Patel, A. Khademhosseini, Effect of biodegradation and de novo matrix synthesis on the mechanical properties of valvular interstitial cell-seeded polyglycerol sebacate-polycaprolactone scaffolds, Acta Biomater. 9 (2013) 5963-5973.

[21] J. Coates, Interpretation of Infrared Spectra, A Practical Approach, Encycl. Anal. Chem. (2006) 1-23. 